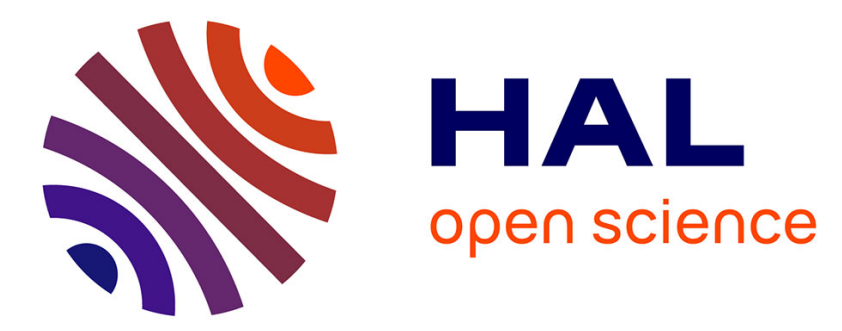

\title{
Point-spread function retrieval for fluorescence microscopy
}

Praveen Pankajakshan, Laure Blanc-Féraud, Zvi Kam, Josiane Zerubia

\section{To cite this version:}

Praveen Pankajakshan, Laure Blanc-Féraud, Zvi Kam, Josiane Zerubia. Point-spread function retrieval for fluorescence microscopy. International Symposium on Biomedical Imaging, Jun 2009, Boston, United States. inria-00395534

\section{HAL Id: inria-00395534 https://hal.inria.fr/inria-00395534}

Submitted on 15 Jun 2009

HAL is a multi-disciplinary open access archive for the deposit and dissemination of scientific research documents, whether they are published or not. The documents may come from teaching and research institutions in France or abroad, or from public or private research centers.
L'archive ouverte pluridisciplinaire HAL, est destinée au dépôt et à la diffusion de documents scientifiques de niveau recherche, publiés ou non, émanant des établissements d'enseignement et de recherche français ou étrangers, des laboratoires publics ou privés. 


\title{
POINT-SPREAD FUNCTION RETRIEVAL FOR FLUORESCENCE MICROSCOPY
}

\author{
Praveen Pankajakshan ${ }^{1}$, Laure Blanc-Féraud ${ }^{1}, Z_{\text {vi Kam }}^{2}$, Josiane \\ Zerubia $^{1}$ \\ ${ }^{1}$ ARIANA Project-team, INRIA/I3S, \\ 2004 route des Lucioles, B.P. 93, \\ 06902 Sophia Antipolis Cedex, France \\ Email: FirstName.LastName@ sophia.inria.fr \\ ${ }^{2}$ Department of Molecular Cell Biology, \\ Weizmann Institute of Science, \\ Rehovot 76100, Israel \\ Email: FirstName.LastName@weizmann.ac.il
}

\begin{abstract}
In this paper we propose a method for retrieving the Point Spread Function (PSF) of an imaging system given the observed image sections of a fluorescent microsphere. Theoretically calculated PSFs often lack the experimental or microscope specific signatures while empirically obtained data are either over sized or (and) too noisy. The effect of noise and the influence of the microsphere size can be mitigated from the experimental data by using a Maximum Likelihood Expectation Maximization (MLEM) algorithm. The true experimental parameters can then be estimated by fitting the result to a model based on the scalar diffraction theory with lower order Spherical Aberration (SA). The algorithm was tested on some simulated data and the results obtained validate the usefulness of the approach for retrieving the PSF from measured data.
\end{abstract}

Index Terms - fluorescence microscopy, point-spread function, Expectation Maximization algorithm, deconvolution, spherical aberration

\section{INTRODUCTION}

In fluorescence microscopy knowledge of the Point-spread function (PSF) is of primary importance as it can be used to accurately reconstruct an object's intensity distribution by deconvolution. Realizing its significance, there have been efforts to theoretically model $[1,2]$ or empirically determine $[3,4]$ and characterize its properties. The classical technique for experimentally determining the PSF is by imaging subresolution fluorescent microsphere. However, since it is very small, the images finally obtained are never an ideal representation of the PSF as they have a low signal-to-noise (SNR) ratio (especially in the far out-of-focus planes). Denoising them can cause loss in essential details and microscope specific information. Theoretically calculated PSFs are popular as they

This research was partially supported by the P2R Franco-Israeli Collaborative Program. The authors gratefully acknowledge Dr. Bo Zhang (Philips Medical research), Prof. Olivier Haeberlé and Prof. Alain Dieterlen (Université de Haute-Alsace, Mulhouse), Prof. Jean-Christophe Olivo-Marin (Pasteur Institute, Paris), and Mr. Gilbert Engler (INRA Sophia-Antipolis) for several interesting discussions. We would also like to thank INRIA for supporting the $\mathrm{PhD}$ of the first author through a CORDI fellowship. do not have the above limitations. They are relevant only when the true experimental parameters can be faithfully determined. Thus, our current proposal is towards retrieving the PSFs from the experimental data by removing the influence of noise and the microsphere size. A constraint on the PSF is introduced to ensure the uniqueness of the solution. This work draws inspiration from our earlier method [5] on the subject of blind deconvolution for diffraction-limited Confocal Laser Scanning Microscopy (CLSM). The difference here is that we are more interested in estimating PSFs that vary with the acquisition conditions; but with some information of the object.

This paper is organized as follows. In Section 2 we introduce the assumptions made in modeling the image formation process and our proposed modification to an existing PSF model to include aberrations. Subsequently, we explain our proposed approach to PSF retrieval from observed data. Section 3 is devoted to implementation details, analysis and results obtained on simulated data. Our conclusions and future perspectives are discussed finally in Section 4.

\section{FRAMEWORK FOR PSF EXTRACTION}

\subsection{Image Formation Model}

Under low illumination and low SNR conditions, when the detector of the fluorescence microscope is behaving as a photon counter (ignoring electronic amplification noise), the number of photons reaching the detector is small and the underlying statistical variation in the number of accumulated photon-electrons can be described by a Poissonian distribution $\mathcal{N}$. Thus if $o$ and $i$ denote the original object and observed image respectively, by ignoring imaging noise, the observation model can be expressed as,

$$
\gamma i(\mathbf{x})=\mathcal{N}(\gamma[(h * o)+b](\mathbf{x})) .
$$

The object's intensity is defined as $\left\{o(\mathbf{x}): \Omega \subset \mathbb{N}^{3} \rightarrow \mathbb{R}\right\}$, where $\Omega$ is the discrete spatial domain over which the intensity is bounded, positive and finite. Here, $h: \Omega \mapsto \mathbb{R}$ is the PSF of the system, $(*)$ denotes the 3-D convolution operator, and $\gamma \in \mathbb{R}^{+}$is the reciprocal of the photon conversion factor [6]. $b: \mathbb{N}^{3} \rightarrow \mathbb{R}$ is a uniformly distributed intensity that models the low-frequency background signal caused by scattered photons and auto-fluorescence from the sample. 


\subsection{Proposed theoretical PSF model}

Before we formulate the model for the PSF, it is important to review the assumptions that will be used in the following section. We will primarily assume that the light used for illuminating the specimen is either circularly polarized or phase randomized. Secondly, from the experiments conducted in the context of our current application, we conclude that vectorial diffraction models offer limited improvement in accuracy than scalar diffraction models (especially for CLSM). This is true even when the difference between the refractive indices of the objective lens and immersion medium is large; like in the case of imaging from air objective lens into specimen embedded in oil or glycerol [6]. We thus justify our usage of scalar models to calculate the PSF as they are computationally less expensive (one integral each for illumination and detection PSF) to calculate than the vectorial models (three integrals for each of the illumination and detection PSF). The model used here is an extension of P. A. Stokseth's [1] work on deriving the Optical Transfer Function (OTF) for a defocused system from the corresponding pupil function. For a microscope with a circular aperture, it is straight forward to show the equivalence between Stokseth's, and Gibson and Lanni's scalar model (see [6]).

The OTF for an incoherent illumination can be obtained from the knowledge of the PSF and vice versa by using the Fourier transform relationship between them. If we consider a converging spherical wave in the object space from the objective lens, the near-focus amplitude distribution $h_{A}$ can be written as $[1,7]$ :

$h_{A}(x, y, z)=\iint_{k_{x}} \int_{k_{y}} P\left(k_{x}, k_{y}, z\right) \exp \left(j\left(k_{x} x+k_{y} y\right)\right) d k_{y} d k_{x}$,

where, $(x, y, z)$ and $\left(k_{x}, k_{y}, k_{z}\right)$ are the $3 \mathrm{D}$ coordinates in the image and the Fourier space respectively, and $j^{2}=-1$. In the above expression, $P\left(k_{x}, k_{y}, z\right)$ describes the overall complex field distribution in the pupil of an objective lens. This pupil function gives a description of the magnitude and phase of the wavefront that a point source produces at the exit pupil of the imaging system. It can be written as [7]:

$$
P= \begin{cases}A\left(\theta_{i}\right) \exp \left(j k_{0} \varphi\left(\theta_{i}, \theta_{s}, z\right)\right), & \text { if } \frac{\sqrt{k_{x}^{2}+k_{y}^{2}}}{k_{i}}<\frac{N A}{n_{i}} \\ 0, & \text { otherwise }\end{cases}
$$

where, $\theta_{i}=\sin ^{-1}\left(k_{x}^{2}+k_{y}^{2}\right)^{1 / 2} / k_{i}$ (see Fig.1), $A\left(\theta_{i}\right)$ is the apodization function $\left(\left(\cos \theta_{i}\right)^{-1 / 2}\right.$ for detection aplanatic lens and $\left(\cos \theta_{i}\right)^{1 / 2}$ for illumination aplanatic lens), $\varphi\left(\theta_{i}, \theta_{s}, z\right)\left(=\varphi_{d}\left(\theta_{i}, z\right)+\varphi_{a}\left(\theta_{i}, \theta_{s}\right)\right)$ is the defocus-aberration phase term, and NA is the numerical aperture of the objective lens. $k_{i}=\left(2 \pi n_{i} / \lambda\right)$, is the wave number of an illumination wave (with a wavelength $\lambda$ in vacuum) in an objective immersion medium. The defocus term $\varphi_{d}$ could be approximated as: $\varphi_{d}\left(\theta_{i}, z\right) \approx k_{i} z\left(1-\cos \theta_{i}\right) / k_{0}$, where $k_{0}=(2 \pi) / \lambda$ is the wave vector in vacuum.

While investigating biological samples, the mounting medium refractive index $n_{s}$ is usually different from that of the objective immersion medium $n_{i}$; especially when imaging live cells in aqueous medium. The observed image is degraded, and the peak amplitude of the PSF is displaced along the optic axis with an asymmetry about the central lateral plane. This is true even at focal depths of a few microns below the cover glass. These degradations are primarily due to an optical effect called Spherical Aberration (SA), where the oblique rays entering a lens are focused differently than the central rays. As this is the dominant aberration observable, the focus of our work will be on retrieving the PSF with only SA. Using simple geometry, it can be shown that the SA phase function is:

$$
\varphi_{a}\left(\theta_{i}, \theta_{s}\right) \approx-d\left(n_{i} \cos \theta_{i}-n_{s} \cos \theta_{s}\right) .
$$

The above phase term relies on the assumption that the error due to mismatch in the refractive indices between the cover glass $n_{g}$ and the objective lens has either been compensated by the correction collar or is minimal. If the cover glass is used with an objective lens that is significantly different than its design specification, an additional phase term should be included, and $d$ replaced by the thickness of the coverslip. Combining (2), (3), and from the Helmholtz reciprocity theo-

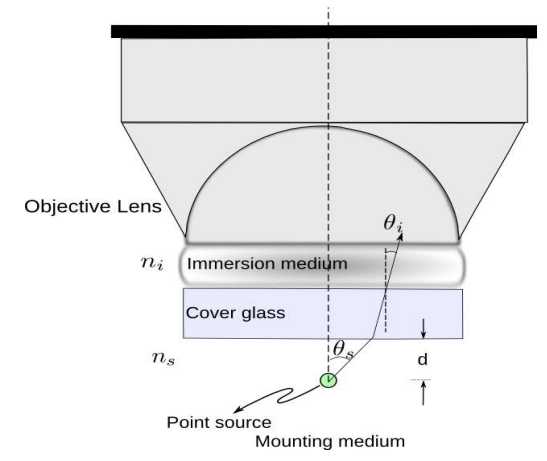

Fig. 1: Schematic showing the imaging of a fluorescent microsphere in a medium of refractive index $n_{s}$ (with $\left.n_{s} \neq n_{i}\right)$.

rem, the CLSM PSF can be written as:

$$
\begin{aligned}
h_{\mathrm{Th}}(\mathbf{x})= & \operatorname{const}\left|h_{A}\left(\mathbf{x} ; \lambda_{e x}\right)\right|^{2} . \\
& \int_{\rho_{1}^{2} \leq \frac{D^{2}}{4}}\left|h_{A}\left(x-x_{1}, y-y_{1}, z ; \lambda_{e m}\right)\right|^{2} d x_{1} d y_{1},
\end{aligned}
$$

where, $\lambda_{e x}$ and $\lambda_{e m}$ is the excitation and the emission wavelengths, $\rho_{1}^{2}=\left(x_{1}^{2}+y_{1}^{2}\right)$, and $D$ is the back-projected diameter of the circular pinhole.

It should be mentioned that for a calibrated CLSM, the variation of the PSF under different imaging conditions is essentially a factor of the following 3 parameters $\vartheta$ : refractive 
index of the objective immersion medium $n_{i}$, index of the specimen or mounting medium $n_{s}$, and depth of the specimen under the cover slip $d$.

\subsection{Proposed approach}

Since the imaged objects are microspheres, their geometry is known a priori and so are their manufacture specified design diameter. If we assume that the observed image $i$ is a realization of an independent Poisson process at each voxel, then the likelihood can be written as:

$$
\operatorname{Pr}(i \mid o, h)=\prod_{\mathbf{x} \in \Omega} \frac{[(h * o)+b](\mathbf{x})^{i(\mathbf{x})} e^{-[(h * o)+b](\mathbf{x})}}{i(\mathbf{x}) !},
$$

where, the mean of the Poisson process is given by $[(h * o)+$ $b](\mathbf{x})$. Maximizing this likelihood (ML) with respect to the PSF gives the following:

$$
\begin{aligned}
\hat{h}_{M L} & =\arg \max _{h}\{\operatorname{Pr}(i \mid o, h)\} \\
& =\arg \min _{h}\{-\log (\operatorname{Pr}(i \mid o, h))\},
\end{aligned}
$$

where, ^represents an estimate. An explicit iterative multiplicative algorithm based on Maximum Likelihood Expectation Maximization (MLEM) [5] formalism can be derived from the above expression as:

$$
\hat{h}_{\mathrm{ML}}^{n+1}(\mathbf{x})=\left(\frac{i(\mathbf{x})}{\left[\left(o * \hat{h}_{\mathrm{ML}}^{n}\right)+\hat{b}\right](\mathbf{x})} * o(-\mathbf{x})\right) \hat{h}_{\mathrm{ML}}^{n}(\mathbf{x}), \forall \mathbf{x} \in \Omega
$$

where, $n$ is the index of iteration of the algorithm. In the above expression, the background fluorescence $b$ is determined as $\hat{b}$ from the smoothed histogram of a single "specimen-independent" slice (see [6]). In (8), the diameter of $o$ is known a priori but its intensity distribution in the medium is unknown. However, it could be assumed to be uniformly distributed with unit intensity while its true value is absorbed into the estimated PSF. The true relative position of the bead $\left(x_{0}, y_{0}, z_{0}\right)$ in the volume should be estimated and the procedure is described in the following section. The algorithm is efficient as it achieves a simultaneous denoisingdeconvolution, and hence ensures that the resulting PSF is free from noise and microsphere size influence. However, the solution space of the PSF is non-unique and hence a form of regularization has to be incorporated into the schema. The estimation process is run for a few iterations (say 30), the resulting PSF $\hat{h}_{\mathrm{ML}}$ is re-projected onto the theoretically modeled PSF space (5) and the parameters $\boldsymbol{\vartheta}=\left\{n_{i}, n_{s}, d\right\}$ are estimated by minimizing the following criterion:

$$
\mathcal{J}(\boldsymbol{\vartheta})=\left\|\hat{h}_{\mathrm{ML}}-h_{\mathrm{Th}}(\boldsymbol{\vartheta})\right\|_{2}^{2}, \forall \boldsymbol{\vartheta} \in \mathbb{R} .
$$

Note that in (9), $\hat{h}_{\mathrm{ML}}$ and $h_{\mathrm{Th}}(\boldsymbol{\vartheta})$ are $\ell_{\infty}$ normalized.

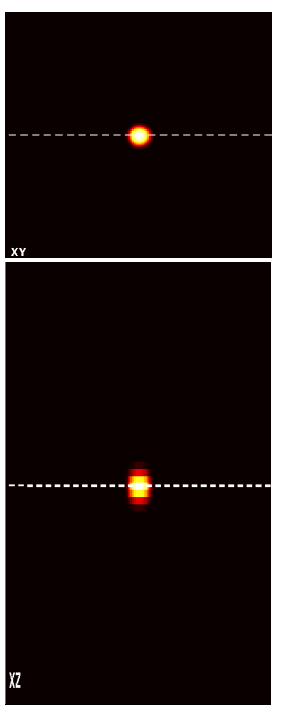

(a)

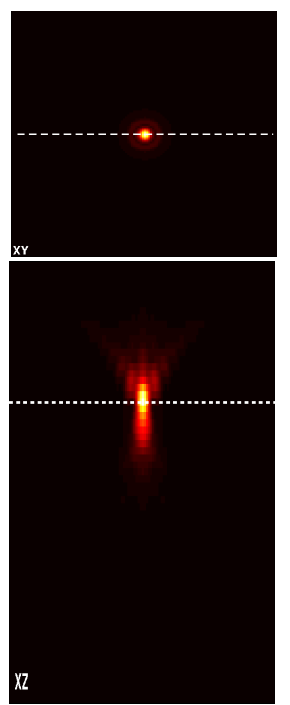

(b)

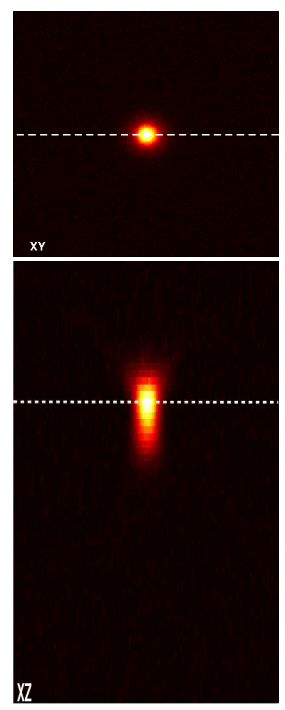

(c)
Fig. 2: Lateral (top) and axial (bottom) sections of the (a) simulated band-limited microsphere, (b) the theoretically modeled PSF (5) at a depth of $10 \mu \mathrm{m}$, and the corresponding (c) blurred-noisy $(\gamma=100)$ simulation of the object as imaged by a CLSM (C) Ariana-INRIA/I3S). The intensity is linearly scaled between $\left[\begin{array}{ll}0 & 1\end{array}\right]$ for display and the dotted lines shows the sections in the volume.

\section{IMPLEMENTATION AND ANALYSIS}

Fig. 1 shows the schematic of imaging a microsphere that is mounted in a medium at a depth $d$. To simulate a bandlimited microsphere, its analytical expression in the frequency domain was multiplied by a $\ell_{\infty}$ normalized 3-D Gaussian function with variances $\left(N_{x}, N_{y}, N_{z}\right) /(2 \pi)$ (see [6]). The radius of the microsphere was chosen to be $250 \mathrm{~nm}$, and it is assumed to be embedded in a medium with $n_{s}=1.33$. The objective lens used is a 'Plan-Neofluar' oil immersion lens $\left(n_{i}=1.518\right)$ with $40 \mathrm{X}$ magnification and Numerical Aperture (NA) of 1.3. The excitation and emission peaks are at wavelengths of $488 \mathrm{~nm}\left(\lambda_{e x}\right)$ and $520 \mathrm{~nm}\left(\lambda_{e m}\right)$ respectively. The physical pinhole size was fixed at $61 \mu \mathrm{m}$, and the images were sampled at lateral and axial pixel sizes of $46.92 \mathrm{~nm}$ and $166.16 \mathrm{~nm}$. Fig. 2 shows the simulated band-limited microsphere, the PSF calculated from (5) at a depth of $10 \mu \mathrm{m}$, and the simulated observation (with $\gamma=100$ ). We denote by $\boldsymbol{\vartheta}_{\text {true }}=\{1.518,1.33,10 \mu m\}$, the true experimental settings of the simulation. The axial profile along the lateral plane is shown in Fig. 3 for a PSF, the blurred and the noisy microsphere.

The initial PSF $\hat{h}_{\mathrm{ML}}^{0}$ for the ML algorithm, is assumed to be constant with the mean of the observed intensities. Fig. 4(b) shows the result of applying the MLEM on the simulated image of a microsphere in Fig. 4(a) after 50 iterations. The microsphere is often not laterally and axially centered so its approximate relative position $\left(x_{0}, y_{0}, z_{0}\right)$ has to be calculated from the observed images. We propose a simple approach in estimating the relative microsphere po- 


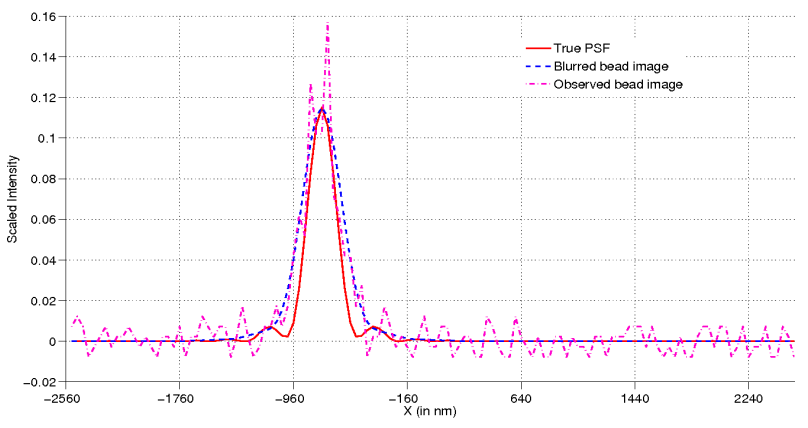

Fig. 3: Axial profiles of a calculated PSF, the blurred microsphere and the observed microsphere. The intensities are scaled and the peaks matched for visual comparison (c) Ariana-INRIA/I3S).

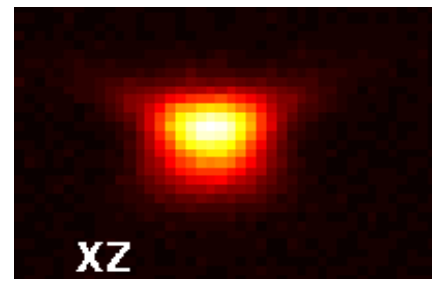

(a)

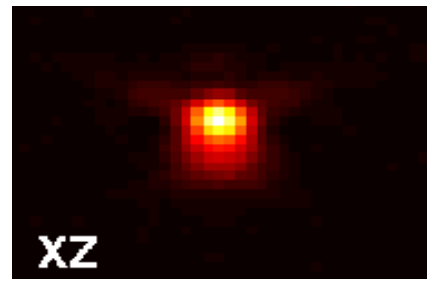

(b)
Fig. 4: Maximum intensity projection along the axial planes of (a) the observed bead and (b) the intermediate MLEM estimated PSF (c) Ariana-INRIA/I3S).

sition in the observed images. The only assumption made here is that the observation data has been treated so that the available data is from a single microsphere. By knowing the physical diameter of the microsphere (here $500 \mathrm{~nm}$ ), we can locate its centroid in the observed volume by estimating the center of gravity (COG) of the intensities [8]. The above method gives very precise lateral locations $\left(\hat{x}_{0}, \hat{y}_{0}\right)$, however, due to focal anomaly the axial location $\left(\hat{z}_{0}\right)$ is inaccurate. From geometrical optics [9], if the objective is non-paraxial, the estimated axial position $\left(\hat{z}_{0}\right)$ is multiplied by a factor $\left(\tan \left(\arcsin \left(N A / n_{i}\right)\right) /\left(\tan \left(\arcsin \left(N A / n_{s}\right)\right)\right)\right.$ to get the new relative position $\left(\hat{z}_{0, \text { new }}\right)$. While for the paraxial case, the multiplication factor becomes $n_{i} / n_{s}$. From simulation results, it was found that this method gives an initial estimate of the relative position to an accuracy of $1.06 \%$ and $0.13 \%$ in the lateral, and $8.42 \%$ in the axial positions respectively. The relative positions estimated using the above technique was used to re-center the object $o$. The most likely estimate of the parameters $\vartheta$ was then found by successive minimization of (9) around an initial guess $\hat{\vartheta}_{0}$. From our experiments it was found that if a bounded estimation approach is used, the final estimates $\hat{\vartheta}$ have a very good correspondence with their true values $\vartheta_{\text {true }}$.

\section{DISCUSSION}

The testing of the algorithm on simulated data shows very promising results for the problem of PSF extraction from observed intensities for a fluorescence microscope where SA is the dominant form of aberration. In the case where subresolution microsphere is used, microsphere size correction will not be necessary and the object should be treated as a Dirac function. Future work is aimed at testing the proposed approach on images of fluorescent polystyrene latex Tetraspeck ${ }^{\mathrm{TM}}$ microspheres from Invitrogen ${ }^{\mathrm{TM}}$. A possible extension of this method might involve estimating the pupil phase without any need for parameter estimation. The task is not simple as there are many possible solutions for the phase function, though a realization might be possible through regularization. This work also opens up new possibilities into the field of depth-varying image restoration.

\section{REFERENCES}

[1] P. A. Stokseth, "Properties of a Defocused Optical System," J. Opt. Soc. Am. A, vol. 59, pp. 1314-1321, Oct. 1969.

[2] S.F. Gibson and F. Lanni, "Diffraction by a circular aperture as a model for three-dimensional optical microscopy," J. Opt. Soc. Am. A, vol. A6, pp. 1357-1367, 1989.

[3] Y. Hiraoka, J. W. Sedat, and D. A. Agard, "Determination of three-dimensional imaging properties of a light microscope system," Biophysical journal, vol. 57, pp. 325-333, Feb. 1990.

[4] J. G. McNally, C. Preza, J.-A. Conchello, and L. J. Thomas, "Artifacts in computational optical-sectioning microscopy," J. Opt. Soc. Am. A, vol. 11, no. 3, pp. 10561067, 1994.

[5] P. Pankajakshan, B. Zhang, L. Blanc-Féraud, Z. Kam, J.C. Olivo-Marin, and J. Zerubia, "Blind deconvolution for diffraction-limited fluorescence microscopy," in Proc. IEEE International Symposium on Biomedical Imaging, Paris, May 2008, pp. 740-743.

[6] P. Pankajakshan, L. Blanc-Féraud, Z. Kam, and J. Zerubia, "Point-Spread Function retrieval for Fluorescence Microscopy," Research Report, INRIA Sophia-Antipolis, France, May 2009.

[7] M. Born and E. Wolf, Principles of Optics, Cambridge Press, Cambridge, 1999.

[8] T. Peng, A. Balijepalli, S. K. Gupta, and T. LeBrun, "Algorithms for On-Line Monitoring of Micro Spheres in an Optical Tweezers-Based Assembly Cell," Journal of Computing and Information Science in Engineering, vol. 7, no. 4, pp. 330-338, 2007.

[9] T. D. Visser, J. L. Oud, and G. J. Brakenhoff, "Refractive index and axial distance measurements in 3-D microscopy," Optik, vol. 90, pp. 17-19, 1992. 